\title{
Case Report \\ Capsule Endoscopy in a Patient with an Implanted CCM System and an Implantable Defibrillator
}

\author{
Florian Streitner, Nina Schoene, Martin Borggrefe, and Jürgen Kuschyk \\ 1st Department of Medicine-Cardiology, University Medical Centre Mannheim, 68167 Mannheim, Germany
}

Correspondence should be addressed to Florian Streitner, florian.streitner@umm.de

Received 2 June 2011; Accepted 27 June 2011

Academic Editors: M.-R. Chen, T. Kasai, R. Steeds, and C. Steinwender

Copyright $\odot 2011$ Florian Streitner et al. This is an open access article distributed under the Creative Commons Attribution License, which permits unrestricted use, distribution, and reproduction in any medium, provided the original work is properly cited.

Wireless video capsule endoscopy (CE) is a modern diagnostic tool. Because of its use of digital radiofrequency, it is still relatively contraindicated in patients with implanted cardiac devices. We report the case of a patient with an Optimizer III system delivering cardiac contractility modulating signals (CCM) for heart failure therapy and an implantable cardioverter defibrillator (ICD) who underwent CE. No interferences between the devices were found.

\section{Introduction}

Wireless video capsule endoscopy (CE) has become the gold standard in patients with unknown blood loss, particularly when there is a high suspicion of small bowel disease [1]. Images acquired by $\mathrm{CE}$ are continuously transmitted by radiofrequency converted into electromagnetic waves to the recorder unit located around the patients waist. Studies to date suggest that $\mathrm{CE}$ is associated with few adverse events $[2,3]$, but the presence of implanted cardiac devices still represents a relative contraindication for several external electromagnetic source applications because of potential interference causing transient or permanent device dysfunction [4]. Therapy with implantable cardioverter-defibrillators (ICDs) itself is well established, whereas cardiac contractility modulation (CCM) represents an upcoming new device therapy developed for heart failure treatment with increasing numbers of devices being implanted worldwide. A CCM device works by stimulating the right ventricular septum with two screw-in leads. The device delivers high-energy stimulation during the absolute refractory period of the heart, thus improving cardiac contractility by influencing calcium fluxes [5].

We report a case of a patient with a CCM System (OPTIMIZER III, Impulse Dynamics USA, Inc., Orangeburg, NY) and a dual-chamber ICD (Atlas + DR V-243, St. Jude Medical, Inc., Sunnyvale, CA) in whom CE (Given PillCam $\mathrm{SB}$, Yoqneam, Israel) was performed. Possible interferences between the three electronic devices were evaluated.

\section{Case Report}

A 74-year-old man was admitted to our hospital because of melena leading to a normochromic normocytic anaemia (haemoglobin on admission $7.5 \mathrm{~g} / \mathrm{dl}, \mathrm{MCV} 91 \mathrm{fl}, \mathrm{MCH}$ $30.7 \mathrm{pg}$, MCHC $33 \mathrm{~g} / \mathrm{dl}$ ). Oral anticoagulation due to mitral valve replacement and intermittent atrial fibrillation was temporarily discontinued. Oesophagogastroduodenoscopy and colonoscopy both gave negative results. As a consequence, $\mathrm{CE}$ was planned to examine the small bowel mucosa despite a concern regarding potential interferences between the radiofrequency emitted by the capsule and the ICD or the CCM device. The CCM device was programmed to deliver therapy 10 hours/day (1 hour CCM delivery, 1.4 hours discontinuation).

After ingesting the PillCam SB capsule, the patient was admitted to the intensive care unit. The CCM and ICD device settings were left unchanged (Table 1). Continuous monitoring of ECG and vital signs was performed. The ICD as well as 
TABLE 1: Device settings.

\begin{tabular}{lc}
\hline \multicolumn{2}{c}{ Optimizer settings } \\
\hline Initiation time & $00: 00$ \\
Termination time & $24: 00$ \\
CCM (hours/day) & 10 \\
CCM signal delay (ms) & 50 \\
CCM signal amplitude (V) & 6.0 \\
CCM signal total duration (ms) & 5.14 \\
Sensitivity RA Lead (mV) & 2 \\
Sensitivity RV Lead (mV) & 2 \\
Sensitivity LS Lead (mV) & 4.1 \\
AV delay (ms) & 252 \\
\hline \multicolumn{1}{c}{ ICD settings } & $60-130$ \\
\hline Pacer programming (DDD/min) & $>150$ \\
VT detection zone (bpm) & $>231$ \\
VF detection zone (bpm) & 310 \\
PVARB (ms) & 250 \\
Ventricular refractory period (ms) & 0.2 (auto) \\
Sensitivity RA Lead (mV) & 0.3 (auto) \\
Sensitivity RV Lead (mV) & $0 / 60$ \\
A/V post-sensed decay delay (ms) & $50 / 62.5$ \\
\hline A/V post-sensed threshold (\%) & RV: \\
\hline
\end{tabular}

CCM: cardiac contractility modulation; RA: right atrium; RV: right ventricle; LS: lower septal; AV: atrioventricular; VT: ventricular tachycardia; VF: ventricular fibrillation; PVARB: postventricular atrial refractory period.

the CCM device were interrogated before, twice during, and directly after the CE procedure. Cross talk tests to exclude oversensing of the ICD leading to inadequate detection of ventricular tachyarrhythmia due to $\mathrm{CE}$ signal transmission or malfunction of the CCM device were performed. During $\mathrm{CE}$ alone or together with CCM delivery, continuous sinus rhythm with a high pacing percentage $(80 \%)$ was noticed. No ventricular tachyarrhythmia or other cardiac adverse events were observed. No short-term artefacts due to CE radiofrequency transmission or due to altered CCM signal delivery were detected during visual online observation of the atrial and ventricular ICD channels (Figures 1 and 2) or during continuous ECG monitoring. No temporary discontinuation or total inactivation of the CCM device was noticed. Active CE did not influence the timing of the atrial triggered ventricular high energy stimulation leading to a safe CCM therapy delivery. Both active cardiac devices did not affect $\mathrm{CE}$ recordings regarding quality or quantity of the transmitted data.

\section{Discussion}

By now, there are no reports regarding $\mathrm{CE}$ in patients with a CCM device, and only a limited number of studies have investigated the potential interference between $\mathrm{CE}$

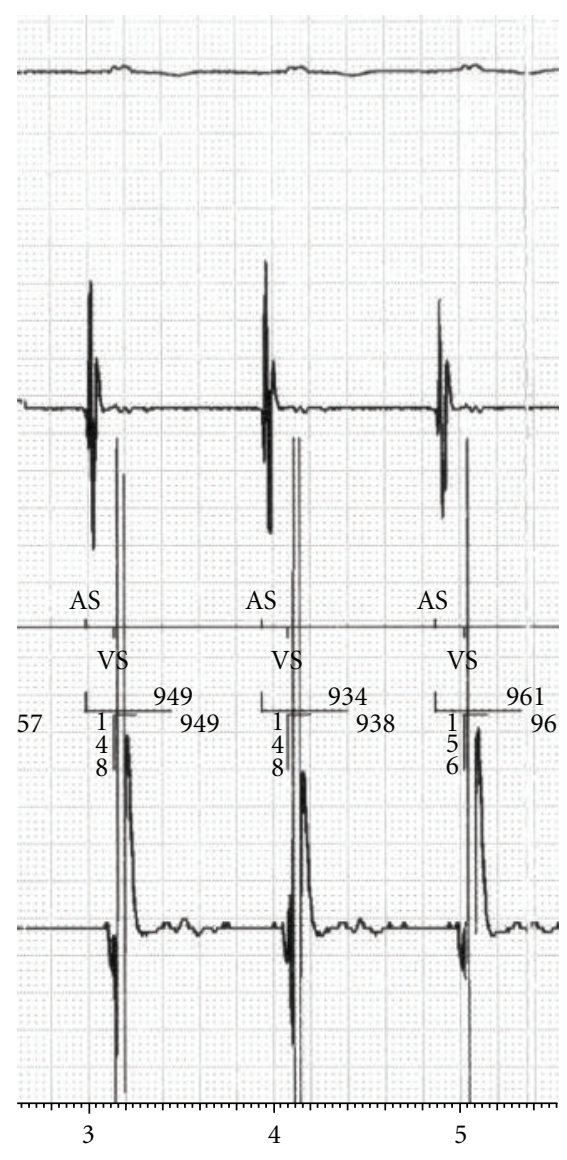

(s)

FIgure 1: ICD Printout: CE, ICD, and CCM active.

and ICDs. Dubner et al. reported an inappropriate shock therapy during in vitro studies in one out of six different tested ICD devices due to electromagnetic interference and raised safety concerns regarding $\mathrm{CE}$ in patients with an ICD [6]. In general, radiofrequency emitted as electromagnetic waves could explain malfunctions of cardiac devices due to a very sensitive setting of special band-pass filters in their sensing circuits. However, the implanted CCM and ICD devices, both using frequencies in the range of $\mathrm{kHz}$ for communication, did not reveal any malfunctions during $\mathrm{CE}$ procedure. Additionally, picture transmission of the PillCam SB to the recorder, which is conducted with a $434.09 \mathrm{MHz}$ carrier frequency and $2 \mathrm{~Hz}$ pulse trains ( $250 \mathrm{~ms}$ on, $250 \mathrm{~ms}$ off) was processed unimpaired leading to clear endoscopic images during the entire recording period.

In summary, no device malfunctions in a patient with an CCM device and an ICD were observed during CE, but because of limited experience, the use of CE in these patients should only be performed in conjunction with close monitoring.

\section{Conflict of Interests}

The authors declare no conflict of interests. 


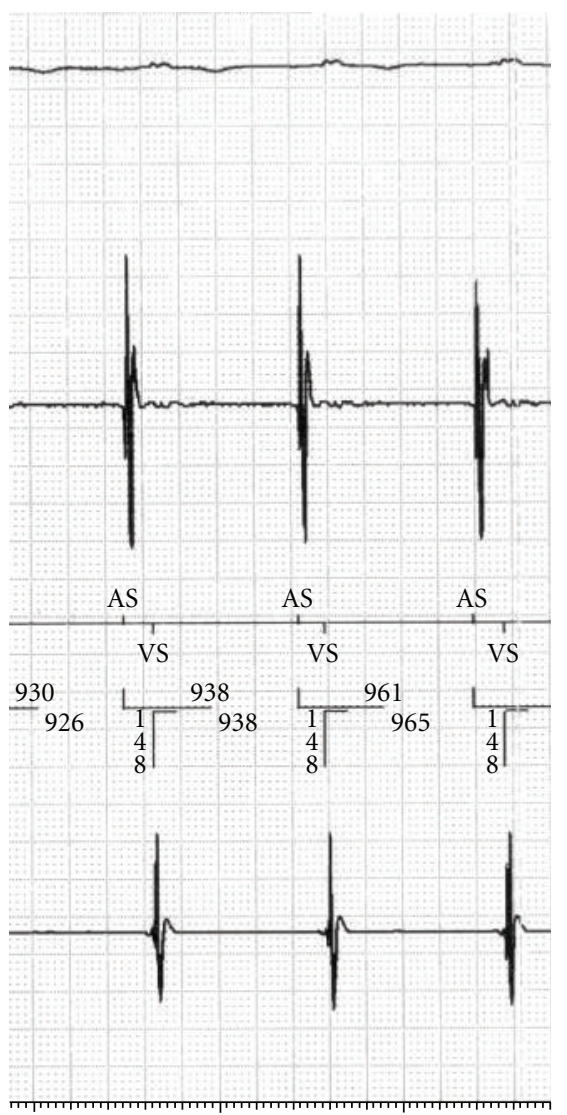

14

15

16

(s)

FIgure 2: ICD Printout: CE and ICD active, CCM off.

\section{References}

[1] G. Iddan, G. Meron, A. Glukhovsky, and P. Swain, "Wireless capsule endoscopy," Nature, vol. 405, no. 6785, pp. 417-418, 2000.

[2] J. A. Leighton, K. Srivathsan, E. J. Carey et al., "Safety of wireless capsule endoscopy in patients with implantable cardiac defibrillators," American Journal of Gastroenterology, vol. 100, no. 8, pp. 1728-1731, 2005.

[3] G. Elias and N. Toubia, "Safety of capsule endoscopy in the setting of implanted cardiac defibrillators: a brief report," American Journal of Gastroenterology, vol. 104, no. 7, pp. 18561857, 2009.

[4] D. L. Hayes, P. J. Wang, D. W. Reynolds et al., "Interference with cardiac pacemakers by cellular telephones," New England Journal of Medicine, vol. 336, no. 21, pp. 1473-1479, 1997.

[5] M. M. Borggrefe, T. Lawo, C. Butter et al., "Randomized, double blind study of non-excitatory, cardiac contractility modulation electrical impulses for symptomatic heart failure," European Heart Journal, vol. 29, no. 8, pp. 1019-1028, 2008.

[6] S. Dubner, Y. Dubner, H. Rubio, and E. Goldin, "Electromagnetic interference from wireless video-capsule endoscopy on implantable cardioverter-defibrillators," Pacing and Clinical Electrophysiology, vol. 30, no. 4, pp. 472-475, 2007. 


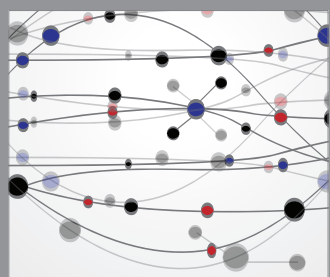

The Scientific World Journal
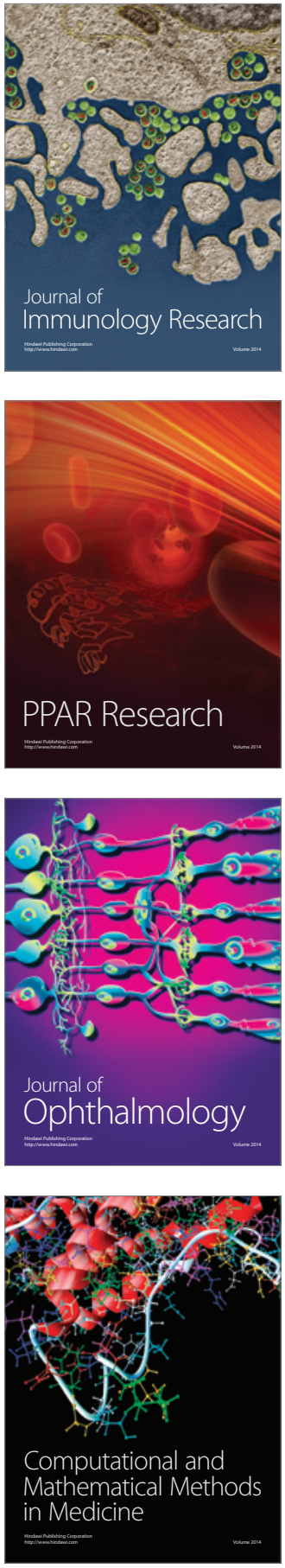

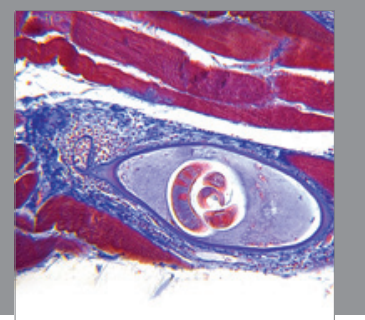

Gastroenterology

Research and Practice
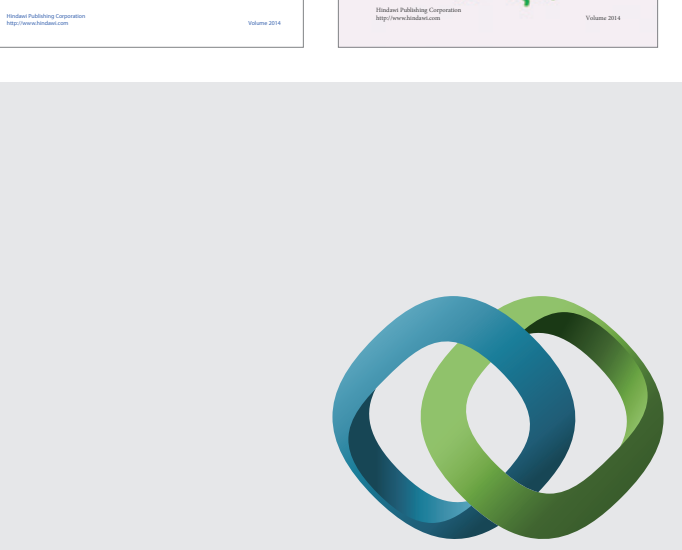

\section{Hindawi}

Submit your manuscripts at

http://www.hindawi.com
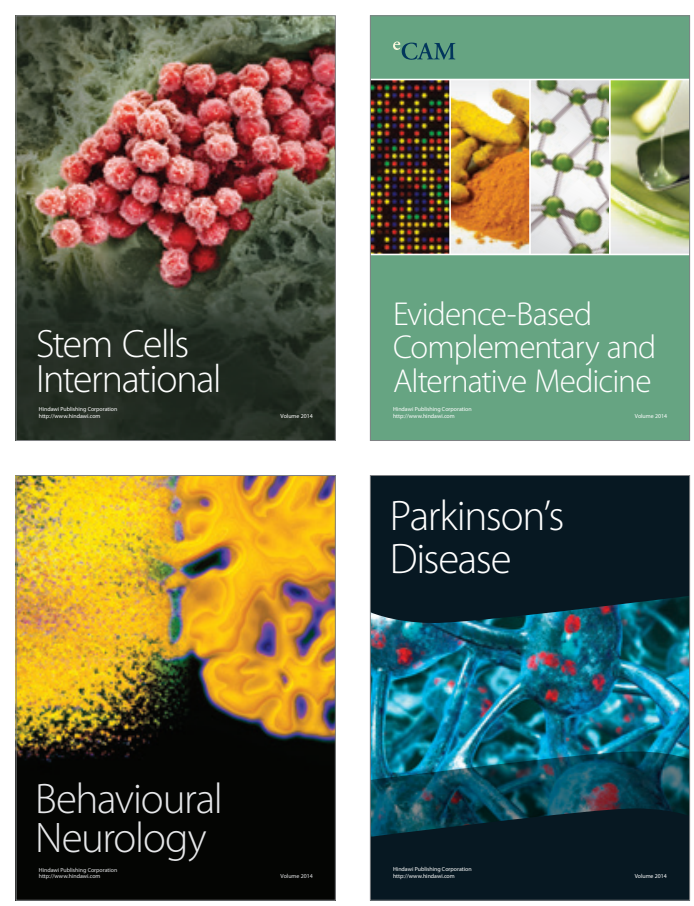

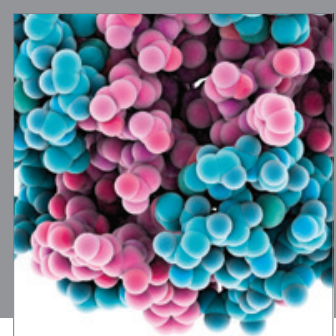

Journal of
Diabetes Research

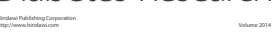

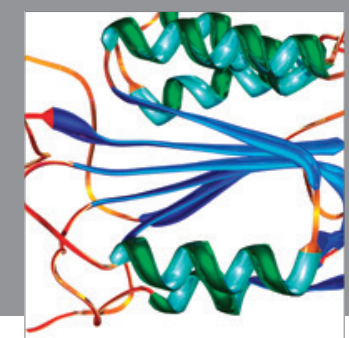

Disease Markers
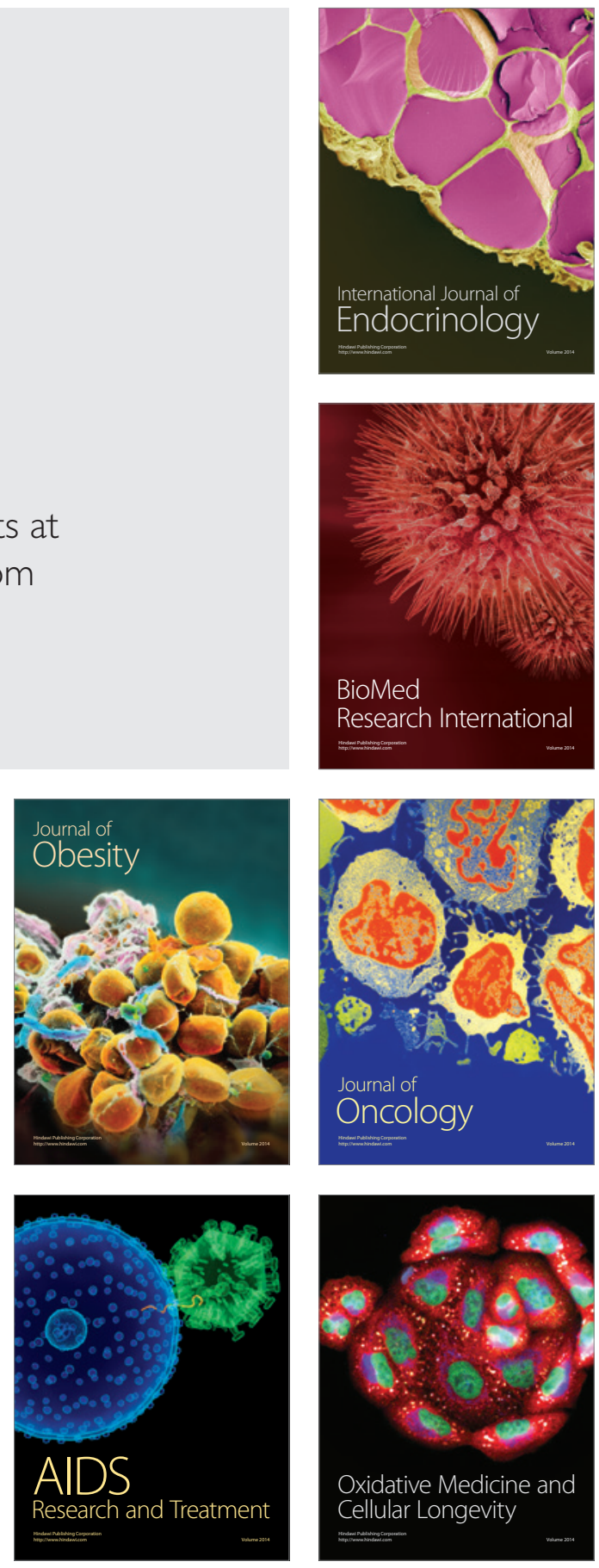